ИЗВЕСТИЯ АКАДЕМИИ НАУК ЭСТОНСКОИ ССР. ФИЗИКА - МАТЕМАТИКА

PROCEEDINGS OF THE ACADEMY OF SCIENCES OF THE ESTONIAN SSR. PHYSICS * MATHEMATICS

$1986,35,1$

\title{
ПРОСВЕТЛЕНИЕ ПОГЛОЩАЮЩИХ ПЛЕНОК
}

\section{Введение}

В настоящей статье мы изложим теорию просветления поглощающих пленок (см. $\left[{ }^{1}\right]$, гл. VIII) в новом виде и с существенными дополнениями. В основу теории положим векторное представление группы пленок (см. $\left[{ }^{1}\right]$, гл. V), ранее использованное лишь в незначительной степени.

Хорошо известно (см. [ $\left.{ }^{1}\right]$, гл. II-III), что всякая интерференционная пленка, состоящая из оптически изотропных слоев, полностью описывается в оптическом отношении матрицей интерференции

$$
F=\left(\begin{array}{ll}
b & \tilde{a}^{*} \\
a & \tilde{b}^{*}
\end{array}\right)
$$

где $a$ и $b$-линейные коэффициенты пленки, связанные с ее амплитудными коэффициентами отражения $r$ и пропускания $t$ формулами

$$
\begin{aligned}
& a=r / t, \\
& b=1 / t .
\end{aligned}
$$

Звездочка означает комплексное сопряжение, а тильдой обозначены величины, относящиеся к сопряженной пленке, т. е. имеющей по сравнению с данной комплексно-сопряженные значения показателей и углов преломления.

Если все слои пленки однородны, что и будем предполагать в дальнейшем, то матрица $F$ выражается формулой

$$
F=G\left(v_{0}\right) L_{1} L_{2} \ldots L_{N} G\left(-v_{N+1}\right)
$$

где $N$ - число слоев, индексы 0 и $N+1$ относятся к ограничивающим средам, а матрицы $L$ суть матрицы интерференции отдельных слоев:

$$
L_{m}=\cos \alpha_{m} \cdot E+i \sin \alpha_{m} \cdot \bar{G}\left(-2 v_{m}\right) .
$$

Матрица $E$ есть единичная матрица, а матрицы $G$ и $\bar{G}$ определяются формулами

$$
G(v)=\left(\begin{array}{lll}
\operatorname{ch} v & \operatorname{sh} v \\
\operatorname{sh} v & \operatorname{ch} v
\end{array}\right)
$$

и

$$
\bar{G}(v)=\left(\begin{array}{ll}
\operatorname{ch} v & -\operatorname{sh} v \\
\operatorname{sh} v & -\operatorname{ch} v
\end{array}\right) .
$$


Величины $v$, называемые рефракторами сред, связаны с показателем преломления $n$ и углом преломления $\vartheta$ формулами

$$
\begin{aligned}
& v^{\mathrm{TE}}=\frac{1}{2} \ln (n \cos \vartheta), \\
& v^{\mathrm{TM}}=\frac{1}{2} \ln \left(n^{-1} \cos \vartheta\right),
\end{aligned}
$$

где индексы TЕ и ТМ различают две возможные линейные поляризации; в случае нормального падения

$$
v=\frac{1}{2} \ln n
$$

Наконец, величины $\alpha$, называемые фазовыми толщинами слоев, определяются формулой

$$
\alpha=k h n \cos \vartheta
$$

где $k$ - волновое число, а $h$ - геометрическая толщина слоя.

Формула (3) имеет эквивалентную форму

$F=G\left(v_{01}\right) M\left(\alpha_{1}\right) G\left(v_{12}\right) M\left(\alpha_{2}\right) \ldots M\left(\alpha_{N-1}\right) G\left(v_{N-1, N}\right) M\left(\alpha_{N}\right) G\left(v_{N, N+1}\right)$,

где

$$
v_{m, m+1}=v_{m}-v_{m+1}
$$

и

$$
M(\alpha)=\left(\begin{array}{cc}
e^{i \alpha} & 0 \\
0 & e^{-i \alpha}
\end{array}\right)
$$

Показатели и углы преломления слоев в общем случае комплексны, но в ограничивающих средах будем считать их вещественными. Представим показатель преломления какого-либо слоя в виде

$$
n=n^{\prime}-i n^{\prime \prime} \text {, }
$$

где $n^{\prime}$ и $n^{\prime \prime}$ неотрицательны. Это значит, что в слое имеет место положительное поглощение, исчезающее только при $n^{\prime \prime \prime}=0$ или $n^{\prime}=0$. Из формулы (13) находим

$$
n \cos \theta=N e^{-\frac{i \theta}{2}}
$$

где

$$
\tan \theta=\frac{2 n^{\prime} n^{\prime \prime}}{n^{\prime 2}-n^{1 / 2}-n_{0}^{2} \sin ^{2} \theta_{0}}, \quad 0 \leqslant \theta \leqslant \pi
$$

и

$$
N=\left[\left(n^{\prime 2}-n^{\prime / 2}-n_{0}^{2} \sin ^{2} \vartheta_{0}\right)^{2}+4 n^{\prime 2} n^{\prime / 2}\right]^{1 / 4} .
$$

Обозначив

$$
\begin{aligned}
& \alpha=\alpha^{\prime}-i \alpha^{\prime \prime}, \\
& v=v^{\prime}-i v^{\prime \prime},
\end{aligned}
$$

имеем согласно формулам (7) и (9):

$$
\begin{aligned}
& \alpha^{\prime}=k h N \cos (\theta / 2) ; \\
& \alpha^{\prime \prime}=k h N \sin (\theta / 2),
\end{aligned}
$$




$$
\begin{aligned}
& v^{\prime \mathrm{TE}}=\frac{1}{2} \ln N, \\
& v^{\prime \mathrm{TM}}=\frac{1}{2} \ln \frac{N}{n^{\prime 2}+n^{\prime / 2}}
\end{aligned}
$$

и

$$
\begin{aligned}
& v^{\prime \prime \mathrm{TE}}=\theta / 4, \\
& v^{\prime \prime \mathrm{TM}}=\theta / 4-\arctan \left(n^{\prime \prime} / n^{\prime}\right) .
\end{aligned}
$$

У сопряженного слоя $n^{\prime \prime}$, $a^{\prime \prime}$ и $v^{\prime \prime}$ имеют обратный знак и поглощение отрицательно. Отсутствие поглощения имеет место в трех случаях: 1) $\left.n^{\prime \prime}=0, n^{\prime}>n_{0} \sin \vartheta_{0} ; 2\right) n^{\prime \prime}=0, n^{\prime}<n_{0} \sin \vartheta_{0}$ (условие полного отражения); 3) $n^{\prime}=0$. В первом случае $\theta, \alpha^{\prime \prime}$ и $v^{\prime \prime}$ обращаются в нуль; во втором случае $\Theta=\pi, \alpha^{\prime}=0$ и $v^{\prime \prime \mathrm{TE}}=v^{\prime \prime \mathrm{TM}}=\frac{\pi}{4} ;$ в третьем случае $\theta=\pi$, $\alpha^{\prime}=0, \quad v^{\prime \prime T E}=\frac{\pi}{4}, \quad v^{\prime \prime T M}=-\frac{\pi}{4}$.

\section{Векторное представление}

Матрица $F$ унимодулярна:

$$
\|F\|=b \tilde{b}^{*}-a \tilde{a}^{*}=1,
$$

так как унимодулярны все матрицы $G, L, M$. Поэтому все возможные матрицы интерференции образуют спинорное представление группы Лоренца. Переход к векторному представлению осуществляется по формуле

$$
\Pi=K\left(P \times P^{*}\right) K^{-1},
$$

где $X$ есть знак прямого произведения, $P$ - любая из матриц $F, G, M$ или $L$, а $\Pi$ - соответствующая ей матрица в векторном представлении, причем

$$
\begin{gathered}
K=\frac{1}{\sqrt{2}}\left[\begin{array}{rrrr}
0 & 1 & 1 & 0 \\
0 & i & -i & 0 \\
1 & 0 & 0 & -1 \\
1 & 0 & 0 & 1
\end{array}\right], \\
K^{-1}=\frac{1}{\sqrt{2}}\left[\begin{array}{rrrr}
0 & 0 & 1 & 1 \\
1 & -i & 0 & 0 \\
1 & i & 0 & 0 \\
0 & 0 & -1 & 1
\end{array}\right] .
\end{gathered}
$$

Все матрицы векторного представления, как и матрицы спинорного представления, унимодулярны. Кроме того, элементы матриц векторного представления удовлетворяют соотношениям ортогональности, согласно формуле

$$
\Pi g \Pi^{\mathrm{T}}=\Pi^{\mathrm{T}} g \Pi=g,
$$

где т - знак транспозиции, а $g$ - матрица метрического тензора пространства-времени:

$$
g=\left[\begin{array}{rrrr}
1 & 0 & 0 & 0 \\
0 & 1 & 0 & 0 \\
0 & 0 & 1 & 0 \\
0 & 0 & 0 & -1
\end{array}\right] .
$$


Главным преимуществом векторного представления является то, что все матрицы в нем вещественны. Вычисляя по формулам (22) и (23) все основные матрицы в векторном представлении, с учетом обозначений (17), находим

$$
\begin{aligned}
& \Gamma(v)=K\left(G(v) \times G\left(v^{*}\right)\right) K^{-1}= \\
& =\left[\begin{array}{cccc}
\operatorname{ch} 2 v^{\prime} & 0 & 0 & \operatorname{sh} 2 v^{\prime} \\
0 & \cos 2 v^{\prime \prime} & -\sin 2 v^{\prime \prime} & 0 \\
0 & \sin 2 v^{\prime \prime} & \cos 2 v^{\prime \prime} & 0 \\
\operatorname{sh} 2 v^{\prime} & 0 & 0 & \operatorname{ch} 2 v^{\prime}
\end{array}\right] \text {, } \\
& \mathrm{M}(\alpha)=K\left(M(\alpha)+M\left(\alpha^{*}\right)\right) K^{-1}= \\
& =\left[\begin{array}{cccc}
\cos 2 \alpha^{\prime} & \sin 2 \alpha^{\prime} & 0 & 0 \\
-\sin 2 \alpha^{\prime} & \cos 2 \alpha^{\prime} & 0 & 0 \\
0 & 0 & \operatorname{ch} 2 \alpha^{\prime \prime} & \operatorname{sh} 2 \alpha^{\prime \prime} \\
0 & 0 & \operatorname{sh} 2 \alpha^{\prime \prime} & \operatorname{ch} 2 \alpha^{\prime \prime}
\end{array}\right] \text {, } \\
& \Lambda=K\left(L \times L^{*}\right) K^{-1}= \\
& =\left[\begin{array}{cc}
\cos 2 \alpha^{\prime} \operatorname{ch}^{2} 2 v^{\prime}- & \sin 2 \alpha^{\prime} \operatorname{ch} 2 v^{\prime} \cos 2 v^{\prime \prime}- \\
-\operatorname{ch} 2 \alpha^{\prime \prime} \operatorname{sh}^{2} 2 v^{\prime} & -\operatorname{sh} 2 \alpha^{\prime \prime} \operatorname{sh} 2 v^{\prime} \sin 2 v^{\prime \prime} \\
-\sin 2 \alpha^{\prime} \operatorname{ch} 2 v^{\prime} \cos 2 v^{\prime \prime}+ & \cos 2 \alpha^{\prime} \cos ^{2} 2 v^{\prime \prime}+ \\
+\operatorname{sh} 2 \alpha^{\prime \prime} \operatorname{sh} 2 v^{\prime} \sin 2 v^{\prime \prime} & +\operatorname{ch} 2 \alpha^{\prime \prime} \sin ^{2} 2 v^{\prime \prime} \\
\sin 2 \alpha^{\prime} \operatorname{ch} 2 v^{\prime} \sin 2 v^{\prime \prime}+ & -\cos 2 \alpha^{\prime} \sin 2 v^{\prime \prime} \cos 2 v^{\prime \prime}+ \\
+\operatorname{sh} 2 \alpha^{\prime \prime} \operatorname{sh} 2 v^{\prime} \cos 2 v^{\prime \prime} & +\operatorname{ch} 2 \alpha^{\prime \prime} \sin 2 v^{\prime \prime} \cos 2 v^{\prime \prime} \\
-\cos 2 \alpha^{\prime} \operatorname{sh} 2 v^{\prime} \operatorname{ch} 2 v^{\prime}+ & -\sin 2 \alpha^{\prime} \operatorname{sh} 2 v^{\prime} \cos 2 v^{\prime \prime}+ \\
+\operatorname{ch} 2 \alpha^{\prime \prime} \operatorname{sh} 2 v^{\prime} \operatorname{ch} 2 v^{\prime} & +\operatorname{sh} 2 \alpha^{\prime \prime} \operatorname{ch} 2 v^{\prime} \sin 2 v^{\prime \prime}
\end{array}\right. \\
& -\sin 2 \alpha^{\prime} \operatorname{ch} 2 v^{\prime} \sin 2 v^{\prime \prime}- \\
& -\operatorname{sh} 2 \alpha^{\prime \prime} \operatorname{sh} 2 v^{\prime} \cos 2 v^{\prime \prime} \\
& -\cos 2 \alpha^{\prime} \sin 2 v^{\prime \prime} \cos 2 v^{\prime \prime}+ \\
& +\operatorname{ch} 2 \alpha^{\prime \prime} \sin 2 v^{\prime \prime} \cos 2 v^{\prime \prime} \\
& \cos 2 \alpha^{\prime} \sin ^{2} 2 v^{\prime \prime}+ \\
& +\operatorname{ch} 2 \alpha^{\prime \prime} \cos ^{2} 2 v^{\prime \prime} \\
& \sin 2 \alpha^{\prime} \operatorname{sh} 2 v^{\prime} \sin 2 v^{\prime \prime}+ \\
& +\operatorname{sh} 2 \alpha^{\prime \prime} \operatorname{ch} 2 v^{\prime} \cos 2 v^{\prime \prime} \\
& \begin{array}{l}
\cos 2 \alpha^{\prime} \operatorname{sh} 2 v^{\prime} \operatorname{ch} 2 v^{\prime}- \\
-\operatorname{ch} 2 \alpha^{\prime \prime} \operatorname{sh} 2 v^{\prime} \operatorname{ch} 2 v^{\prime} \\
-\sin 2 \alpha^{\prime} \operatorname{sh} 2 v^{\prime} \cos 2 v^{\prime \prime}+ \\
+\operatorname{sh} 2 \alpha^{\prime \prime} \operatorname{ch} 2 v^{\prime} \sin 2 v^{\prime \prime} \\
\sin 2 \alpha^{\prime} \operatorname{sh} 2 v^{\prime} \sin 2 v^{\prime \prime}+ \\
+\operatorname{sh} 2 \alpha^{\prime \prime} \operatorname{ch} 2 v^{\prime} \cos 2 v^{\prime \prime} \\
-\cos 2 \alpha^{\prime} \operatorname{sh}^{2} 2 v^{\prime}+ \\
+\operatorname{ch} 2 \alpha^{\prime \prime} \operatorname{ch}^{2} 2 v^{\prime}
\end{array}
\end{aligned}
$$

и

$$
\begin{aligned}
& \Phi=K\left(F \times F^{*}\right) K^{-1}= \\
& =\frac{1}{2}\left[\begin{array}{cc}
b \tilde{b}+b^{*} \tilde{b}^{*}+a \tilde{a}+a^{*} \tilde{a}^{*} & i\left(-b \tilde{b}+b^{*} \tilde{b}^{*}-a \tilde{a}+a^{*} \tilde{a}^{*}\right) \\
i\left(b \tilde{b}-b^{*} \tilde{b}^{*}-a \tilde{a}+a^{*} \tilde{a}^{*}\right) & b \tilde{b}+b^{*} \tilde{b}^{*}-a \tilde{a}-a^{*} \tilde{a}^{*} \\
\tilde{a} b-a \tilde{b}+\tilde{a}^{*} b^{*}-a^{*} \tilde{b}^{*} & i\left(-\tilde{a} b+a \tilde{b}+\tilde{a}^{*} b^{*}-a^{*} \tilde{b}^{*}\right) \\
\tilde{a} b+a \tilde{b}+\tilde{a}^{*} b^{*}+a^{*} \tilde{b}^{*} & i\left(-\tilde{a} b-a \tilde{b}+\tilde{a}^{*} b^{*}+a^{*} \tilde{b}^{*}\right)
\end{array}\right. \\
& a^{*} b-\tilde{a}^{*} \tilde{b}+a b^{*}-\tilde{a} \tilde{b}^{*} \\
& i\left(a^{*} b-\tilde{a}^{*} \tilde{b}-a b^{*}+\tilde{a} \tilde{b}^{*}\right)
\end{aligned}
$$

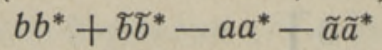

$$
\begin{aligned}
& b b^{*}-\tilde{b} \tilde{b}^{*}+a a^{*}-\tilde{a} \tilde{a}^{*}
\end{aligned}
$$

Последнюю формулу перепишем еще в ином виде, введя следующие обозначения: 


$$
\begin{gathered}
b \tilde{b}-a \tilde{a}=X e^{i \xi}, \\
b \tilde{b}+a \tilde{a}=Y e^{i \eta}, \\
\tilde{a} b-a \tilde{b}=U e^{i \sigma}, \\
\tilde{a} b+a \tilde{b}=V e^{i \tau}, \\
\frac{1}{2}\left(b b^{*}+\tilde{b} \tilde{b}^{*}+a a^{*}+\tilde{a} \tilde{a}^{*}\right)=\operatorname{ch} \psi, \\
\frac{1}{2}\left(b b^{*}+\tilde{b} \tilde{b}^{*}-a a^{*}-\tilde{a} \tilde{a}^{*}\right)=\operatorname{ch} \varphi, \\
\frac{1}{2}\left(b b^{*}-\tilde{b} \tilde{b}^{*}-a a^{*}+\tilde{a} \tilde{a}^{*}\right)=S .
\end{gathered}
$$

Учтя вдобавок формулы (см. [1], с. 29)

$$
\begin{aligned}
& a^{\prime}=-\tilde{a}^{*},- \\
& b^{\prime}=b,
\end{aligned}
$$

где штрих означает падение света на пленку в обратном направлении, получаем

$$
\Phi=\left[\begin{array}{cccc}
Y \cos \eta & Y \sin \eta & -U^{\prime} \cos \sigma^{\prime} & -V^{\prime} \cos \tau^{\prime} \\
-X \sin \xi & X \cos \xi & U^{\prime} \sin \sigma^{\prime} & V^{\prime} \sin \tau^{\prime} \\
U \cos \sigma & U \sin \sigma & \operatorname{ch} \varphi & S \\
V \cos \tau & V \sin \tau & S^{\prime} & \operatorname{ch} \psi
\end{array}\right] .
$$

Отметим, что, как показано в $\left[{ }^{1}\right]$ (см. $\left.§ 16\right)$, если поглощение во всех слоях пленки положительно, то $S>0$ и $\varphi$ вещественно. Это мы и будем предполагать.

Рассмотрим частный случай отсутствия поглощения. Из выводов в конце предыдущего раздела следует, что если $n^{\prime \prime}=0$ и $n^{\prime}>n_{0} \sin \vartheta_{0}$, то

$$
\begin{aligned}
\operatorname{sh} 2 \alpha^{\prime \prime}= & \sin 2 v^{\prime \prime}=0, \\
\cos 2 v^{\prime \prime} & =1,
\end{aligned}
$$

а если $n^{\prime \prime}=0$ и $n^{\prime}<n_{0} \sin \vartheta_{0}$ или $n^{\prime}=0$, то

$$
\begin{aligned}
\sin 2 \alpha^{\prime}= & \cos 2 v^{\prime \prime}=0, \\
\cos 2 \alpha^{\prime} & =1 .
\end{aligned}
$$

В обоих случаях, согласно формуле (28), элементы третьей строки и третьего столбца матрицы $\Lambda$ обращаются в нуль, кроме элемента $\Lambda_{33}=1$. То же свойство имеют и матрицы $\Gamma\left(v_{0}\right)$ и $\Gamma\left(-v_{N+1}\right)$, так как мы приняли, что углы падения и преломления в ограничивающих средах вещественны. Следовательно, если поглощение отсутствует во всех слоях, то и матрица

$$
\Phi=\Gamma\left(v_{0}\right) \Lambda_{1} \Lambda_{2} \ldots \Lambda_{N} \Gamma\left(-v_{N+1}\right)
$$

обладает тем же свойством. Четырехрядные матрицы векторного представления обращаются, таким образом, в трехрядные. Согласно формулам (35), (36) и (38), при отсутствии в пленке поглощения

$$
\begin{gathered}
\operatorname{ch} \varphi=1, \\
S=S^{\prime}=U=U^{\prime}=0,
\end{gathered}
$$

откуда $S+\operatorname{ch} \varphi=1$, т. е.

$$
b b^{*}-a a^{*}=1,
$$


как и должно быть. Это равенство равносильноо соотношению

$$
R+T=1 \text {, }
$$

где $R=a a^{*} / b b^{*}$ - энергетический коэффициент отражения и $T=$ $=1 / b b^{*}$ - энергетический коэффициент пропускания. Далее, из равенства $U=0$ по формуле (32) имеем

$$
\tilde{a} b-a \tilde{b}=0 .
$$

Отсюда и из формул (21) и (43) следует

$$
\begin{aligned}
& \tilde{a}=a, \\
& \tilde{b}=b .
\end{aligned}
$$

Подставляя $a$ и $b$ вместо $\tilde{a}$ и $\tilde{b}$ в формулу (29), находим для матрицы интерференции непоглощающей пленки формулу

$$
\Phi=\left[\begin{array}{cccc}
\frac{1}{2}\left(b^{2}+b^{* 2}+\right. & \frac{i}{2}\left(-b^{2}+b^{* 2}-\right. & 0 & a^{*} b+a b^{*} \\
\left.+a^{2}+a^{* 2}\right) & \left.-a^{2}+a^{* 2}\right) & & \\
\frac{i}{2}\left(b^{2}-b^{* 2}-\right. & \frac{1}{2}\left(b^{2}+b^{* 2}-\right. & 0 & i\left(a^{*} b+a b^{*}\right) \\
\left.-a^{2}+a^{* 2}\right) & \left.-a^{2}-a^{* 2}\right) & & \\
0 & 0 & 1 & 0 \\
a b+a^{*} b^{*} & i\left(-a b+a^{*} b^{*}\right) & 0 & b b^{*}+a a^{*}
\end{array}\right] .
$$

Если обозначим фазы линейных коэффициентов пленки через $\mu$ и $v$, так что

$$
\begin{aligned}
& a=\sqrt{R / T} e^{i \mu}, \\
& b=\frac{e^{i \nu}}{\sqrt{T}},
\end{aligned}
$$

то это выражение примет вид

$$
\Phi=\frac{1}{T}\left[\begin{array}{cccc}
\cos 2 v+R \cos 2 \mu & \sin 2 v+R \sin 2 \mu & 0 & 2 \sqrt{R} \cos (\mu-v) \\
-\sin 2 v+R \sin 2 \mu & \cos 2 v-R \cos 2 \mu & 0 & 2 \sqrt{R} \sin (\mu-v) \\
0 & 0 & 1-R & 0 \\
2 \sqrt{R} \cos (\mu+v) & 2 \sqrt{R} \sin (\mu+v) & 0 & 1+R
\end{array}\right] .
$$

\section{Просветление}

Всякая поглощающая пленка имеет следующие свойства:

1. Характеризующая поглощающую пленку величина $\varphi$ (см. формулы (35) и (38)) не изменяется, если к этой пленке добавляются с обеих сторон непоглощающие пленки.

Для доказательства обозначим матрицу интерференции поглощающей пленки, как и ранее, через $\Phi$, а матрицы интерференции добавляемых непоглощающих пленок через $\Phi_{0}$ (спереди) и $\Phi_{a}$ (сзади). Тогда матрица интерференции всей системы выразится как произведение 
$\Phi_{0} \Phi \Phi_{a}$. Так как матрицы $\Phi_{0}$ и $\Phi_{a}$ имеют в третьем ряду и третьем столбце нули, кроме равного единице элемента на их пересечении, то

$$
\left(\Phi_{0} \Phi \Phi_{a}\right)_{33}=\Phi_{33}
$$

что и требовалось показать.

2. Характеризующие поглощающую пленку величины $U, \sigma$ и $S$ (см. формулы (32), (36) и (38)) не изменяются, если к этой пленке добавляется спереди непоглощающая пленка. Равным образом, не изменяются величины $U^{\prime}, \sigma^{\prime}, S^{\prime}$, если к пленке добавляется непоглощающая пленка сзади.

Доказательство вытекает из того же свойства непоглощающих пленок, в силу которого

$$
\begin{aligned}
& \left(\Phi_{0} \Phi\right)_{3 \mu}=\Phi_{3 \mu}, \\
& \left(\Phi \Phi_{a}\right)_{\mu s}=\Phi_{\mu 3},
\end{aligned}
$$

что и требовалось показать.

3. Если неполлощающая пленка добавляется к поглощающей пленке сзади, то элементы третьего ряда матрицы интерференции $U \cos \sigma$, $U \sin \sigma$ и $S$ изменяются. При надлежащем выборе добавочной непоглощающей пленки $U$ обращается в нуль. Это и называется просветлением. Обоснование этого названия будет дано ниже.

Выясним условия просветления. Величины, относящиеся к непросветленной поглощающей пленке, будем писать без индекса, а величины, относящиеся к просветленной и просветляющей пленкам, обозначим индексами 1 и $a$ соответственно. Таким образом,

$$
\Phi_{1}=\Phi \Phi_{a}
$$

Вычисляя элементы третьего ряда этого произведения по формулам (38) и (49) и приравнивая нулю два первых элемента (т. е. полагая $\left.U_{1}=0\right)$, находим

$$
\begin{gathered}
U \cos \left(2 v_{a}+\sigma\right)+U R_{a} \cos \left(2 \mu_{a}-\sigma\right)+2 S \sqrt{R_{a}} \cos \left(\mu_{a}+v_{a}\right)=0 \\
U \sin \left(2 v_{a}+\sigma\right)+U R_{a} \sin \left(2 \mu_{a}-\sigma\right)+2 S \sqrt{R_{a}} \sin \left(\mu_{a}+v_{a}\right)=0 \\
\operatorname{ch} \varphi_{1}=\operatorname{ch} \varphi
\end{gathered}
$$

и

$$
S_{1}=\frac{\left(1+R_{a}\right) S+2 U \sqrt{R_{a}} \cos \left(\mu_{a}-v_{a}-\sigma\right)}{1-R_{a}} .
$$

Формула (55) уже известна по (50). Объединяя формулы (53) и (54), получаем для $\sqrt{R_{a}}$ квадратное уравнение

$$
U e^{i\left(2 \mu_{a}-\sigma\right)} R_{a}+2 S e^{i\left(\mu_{a}+v_{a}\right)} \sqrt{R_{a}}+U e^{i\left(2 v_{a}+\sigma\right)}=0,
$$

решая которое, находим

$$
\sqrt{R_{a}}=\frac{e^{i\left(-\mu_{a}+v_{a}+\sigma\right)}\left(-S \pm \sqrt{S^{2}-U^{2}}\right)}{U}
$$

Здесь $S$ и $U$ положительны, причем, в силу ортогональности матрицы $\Phi$,

$$
S^{2}-U^{2}=\operatorname{sh}^{2} \varphi
$$


Следовательно,

$$
\sqrt{R_{a}}=-e^{i\left(-\mu_{a}+v_{a}+\sigma\right)} \sqrt{\frac{S \mp \operatorname{sh} \varphi}{S_{ \pm} \operatorname{sh} \varphi}} .
$$

Так как $\sqrt{R_{a}}$ вещественно и $0 \leqslant \sqrt{R_{a}}<1$, то

$$
\cos \left(\sigma-\mu_{a}+v_{a}\right)=-1,
$$

а под корнем следует взять верхний знак:

$$
R_{a}=\frac{S-\operatorname{sh} \varphi}{S+\operatorname{sh} \varphi} .
$$

Формулы (61) и (62) являются в совокупности условием просветления. Если они удовлетворяются, то

$$
U_{1}=0,
$$

откуда, в силу ортогональности матрицы $Ф \Phi_{a}$, следует

$$
S_{1}=\operatorname{sh} \varphi .
$$

В этом можно убедиться иначе, подставляя выражения $\cos \left(\sigma-\mu_{a}+v_{a}\right)$ и $R_{a}$ из формул $(61)$ и $(62)$ в формулу (56).

Итак, в результате просветления элементы третьего ряда матрицы интерференции пленки получают значения $0,0 \mathrm{ch} \varphi$, sh $\varphi$. Элементы третьего столбща при просветлении не изменяются вообще (см. вторую формулу (51)). Посмотрим, как изменяются остальные элементы.

Элементы четвертого столбца найти проще всего, используя ортогональность матрицы $\Phi_{1}$ (см. формулу (24)). Во-первых,

$$
\left(\Phi_{1} g \Phi_{1}^{\mathrm{T}}\right)_{34}=0,
$$

т. е., согласно формулам (63) и (64),

$$
S^{\prime} \operatorname{ch} \varphi-\operatorname{sh} \varphi \operatorname{ch} \psi_{1}=0 .
$$

Отсюда

$$
\operatorname{ch} \psi_{1}=\Phi_{1,44}=S^{\prime} \operatorname{cth} \varphi
$$

Далее,

$$
\left(\Phi_{1}^{\mathrm{T}} g \Phi_{1}\right)_{44}=-1,
$$

т. е., согласно формулам (64) и (67),

$$
V_{1}^{\prime 2}+\operatorname{sh}^{2} \varphi-S^{\prime 2} \operatorname{cth}^{2} \varphi=-1,
$$

откуда

$$
V_{1}^{\prime}=U^{\prime} \operatorname{cth} \varphi
$$

Наконец,

$$
\left(\Phi_{1}^{\mathrm{T}} g \Phi_{1}\right)_{34}=0,
$$

т. е., согласно формулам (64), (67) и (70),

$$
U^{\prime 2} \operatorname{cth} \varphi \cos \left(\sigma^{\prime}-\tau_{1}^{\prime}\right)+\operatorname{sh} \varphi \operatorname{ch} \varphi-S^{\prime 2} \operatorname{cth} \varphi=0,
$$

откуда

$$
U^{\prime 2}\left(1-\cos \left(\sigma^{\prime}-\tau_{1}^{\prime}\right)\right)=0 .
$$

Если $U^{\prime} \neq 0$, то отсюда следует $\tau_{1}^{\prime}=\sigma^{\prime}$; если же $U^{\prime}=0$, то $\sigma^{\prime}$ произ- 
вольн̈о, а так как тогда, в силу формулы $(70), V_{1}^{\prime}=0$, то и $\tau^{\prime}{ }_{1}$ пройзвольно. Поэтому в любом случае

$$
\tau_{1}^{\prime}=\sigma^{\prime}
$$

Отсюда и из формулы (70) следует

$$
\begin{aligned}
& \Phi_{1,14}=-V_{1}^{\prime} \cos \tau_{1}^{\prime}=-U^{\prime} \cos \sigma^{\prime} \operatorname{cth} \varphi, \\
& \Phi_{1,24}=V_{1}^{\prime} \sin \tau_{1}^{\prime}=U^{\prime} \sin \sigma^{\prime} \operatorname{cth} \varphi .
\end{aligned}
$$

Итак, матрицу интерференции $\Phi_{1}$ просветленной поглощающей пленки можно записать в виде

$$
\Phi_{1}=\left[\begin{array}{cccc}
Y_{1} \cos \eta_{1} & Y_{1} \sin \eta_{1} & -U^{\prime} \cos \sigma^{\prime} & -U^{\prime} \cos \sigma^{\prime} \operatorname{cth} \varphi \\
-X_{1} \sin \xi_{1} & X_{1} \cos \xi_{1} & U^{\prime} \sin \sigma^{\prime} & U^{\prime} \sin \sigma^{\prime} \operatorname{cth} \varphi \\
0 & 0 & \operatorname{ch} \varphi & \operatorname{sh} \varphi \\
V_{1} \cos \tau_{1} & V_{1} \sin \tau_{1} & S^{\prime} & S^{\prime} \operatorname{cth} \varphi
\end{array}\right]
$$

Элементы 1-го и 2-го столбцов здесь пока только обозначены. Дело в том, что эти элементы одними лишь условиями просветления (61) и (62) однозначно не определяются. Однако мы можем дать им выражение, в котором условия просветления в меру возможности учитываются. Для этого прежде всего докажем соотношения

$$
\begin{aligned}
& \tilde{a}_{1}=a_{1} e^{-\varphi,} \\
& \tilde{b}_{1}=b_{1} e^{-\varphi},
\end{aligned}
$$

связывающие линейные коэффициенты просветленной пленки. Для доказательства напишем формулы

$$
\begin{aligned}
& \tilde{a}_{1} b_{1}-a_{1} \widetilde{b}_{1}=0, \\
& b_{1} \widetilde{b}_{1}^{*}-a_{1} a_{1}^{*}=e^{\varphi},
\end{aligned}
$$

вытекающие, согласно формулам (32), (35) и (36), из третьего ряда матрицы $\Phi_{1}$. Добавим формулу (см. 21))

$$
b_{1}^{*} \tilde{b}_{1}-a_{1}^{*} \tilde{a}_{1}=1 \text {. }
$$

Выражая из первой формулы (78) и формулы (79) $\tilde{a}_{1}$ и $\tilde{b}_{1}$, находим

$$
\begin{aligned}
& \tilde{a}_{1}=a_{1}\left(b_{1} b_{1}^{*}-a_{1} a_{1}^{*}\right)^{-1}, \\
& \tilde{b}_{1}=b_{1}\left(b_{1} b_{1}^{*}-a_{1} a_{1}^{*}\right)^{-1},
\end{aligned}
$$

откуда, в силу второй формулы (78), и вытекают соотношения (77).

Обозначим фазы линейных коэффициентов $a_{1}$ и $b_{1}$ через $\mu_{1}$ и $v_{1}$ :

$$
\begin{aligned}
& a_{1}=\sqrt{R_{1} / T_{1}} e^{i \mu_{1}}, \\
& b_{1}=\frac{e^{i v_{1}}}{\sqrt{T_{1}}},
\end{aligned}
$$

где $R_{1}$ и $T_{1}-$ энергетические коэффициенты отражения и пропускания просветленной пленки. Из формул (77) следует

$$
\begin{aligned}
& \tilde{a}_{1}=\sqrt{R_{1} / T_{1}} e^{i \mu_{1}} e^{-\Phi}, \\
& b_{1}=\frac{e^{i v_{1}} e^{-\varphi}}{\sqrt{T_{1}}} .
\end{aligned}
$$


Подставляя выражения (81) и (82) в элементы матрицы $\Phi_{1}$ (взятой в виде (29)), находим ее в виде .

$\Phi_{1}=\frac{e^{-\varphi}}{T} \times$

$\times\left[\begin{array}{cccc}\cos 2 v_{1}+ & \sin 2 v_{1}+ & 2 \sqrt{R_{1}} \cos \left(\mu_{1}-v_{1}\right) \operatorname{sh} \varphi & 2 \sqrt{R_{1}} \cos \left(\mu_{1}-v_{1}\right) \operatorname{ch} \varphi \\ +R_{1} \cos 2 \mu_{1} & +R_{1} \sin 2 \mu_{1} & & \\ -\sin 2 v_{1}+ & \cos 2 v_{1}- & 2 \sqrt{R_{1}} \sin \left(\mu_{1}-v_{1}\right) \operatorname{sh} \varphi & 2 \sqrt{R_{1}} \sin \left(\mu_{1}-v_{1}\right) \operatorname{ch} \varphi \\ +R_{1} \sin 2 \mu_{1} & -R_{1} \cos 2 \mu_{1} & \left(1-R_{1}\right) \operatorname{ch} \varphi & \left(1-R_{1}\right) \operatorname{sh} \varphi \\ 0 & 0 & \left(1+R_{1}\right) \operatorname{sh} \varphi & \left(1+R_{1}\right) \operatorname{ch} \varphi\end{array}\right]$.

Эта формула эквивалентна формуле (76). Приравнивая элементы третьего столбца обеих формул, находим

$$
\begin{gathered}
\cos \left(\mu_{1}-v_{1}\right)=-\cos \sigma^{\prime}, \\
\sin \left(\mu_{1}-v_{1}\right)=\sin \sigma^{\prime}, \\
\frac{2 \sqrt{R_{1}}}{T_{1}}=\frac{U^{\prime} e^{\varphi}}{\operatorname{sh} \varphi}, \\
\frac{1-R_{1}}{T_{1}}=e^{\varphi}
\end{gathered}
$$

и

$$
\frac{1+R_{1}}{T_{1}}=\frac{S^{\prime} e^{\varphi}}{\operatorname{sh} \varphi}
$$

Из формул (84) следует

$$
\cos \left(\mu_{1}-v_{1}+\sigma^{\prime}\right)=-1,
$$

а из формул $(85)-(87)$ находим энергетические коэффициенты просветленной пленки, выраженные через характеристические величины $S^{\prime}$ и $\varphi:$

$$
\begin{aligned}
& R_{1}=\frac{S^{\prime}-\operatorname{sh} \varphi}{S^{\prime}+\operatorname{sh} \varphi}, \\
& T_{1}=\frac{1-e^{-2 \varphi}}{S^{\prime}+\operatorname{sh} \varphi} \\
& A_{1}=\frac{2 \operatorname{sh} \varphi\left(1-e^{-\varphi}\right)}{S^{\prime}+\operatorname{sh} \varphi}
\end{aligned}
$$

причем

$$
\frac{A_{1}}{T_{1}}=e^{\varphi}-1
$$

Последняя формула показывает, что отношение $A_{1} / T_{1}$ имеет у просветленной пленки наименьшее возможное значение, чем и оправдывается название просветления. В самом деле, из формул (29) и (38) следует

$$
\frac{A}{T}=\operatorname{ch} \varphi+S-1 \text {. }
$$


$\widehat{\mathrm{B}}$ силу соотношения (59) $\hat{S}$ имеет наименьшее значение при $\dot{U}=0$, т. ё. при наличии просветления. Тогда $S=\operatorname{sh} \varphi$ и формула (91) получает вид (90).

Интересно отметить, что матрица интерференции просветленной пленки обнаруживает близкое сходство с матрицей интерференции непоглощающей пленки (см. формулу (49)). Обозначив $\Phi_{1}$ при $\varphi=0$ через $\Phi_{1}{ }^{0}$, находим

$$
\Phi_{1}=e^{-\Phi} \Phi_{1}^{0}(E \oplus G(\varphi)),
$$

где $\oplus-$ знак прямой суммы.

Если пленка просветлена с обеих сторон, то матрица интерференции сильно упрощается. Обозначая величины, относящиеся к двусторонне просветленной пленке, индексом 2 , имеем прежде всего

$$
a_{2}=0 \text {, }
$$

что вытекает из первых формул (37) и (77). Следовательно,

$$
R_{2}=0 \text {. }
$$

Тогда $A_{2}+T_{2}=1$, а так как $A_{2} / T_{2}=e^{\varphi}-1$, то

$$
T_{2}^{-1} e^{-\varphi}=1
$$

и матрица интерференции принимает вид

$$
\Phi_{2}=\left[\begin{array}{cccc}
\cos 2 v_{2} & \sin 2 v_{2} & 0 & 0 \\
-\sin 2 v_{2} & \cos 2 v_{2} & 0 & 0 \\
0 & 0 & \operatorname{ch} \varphi & \operatorname{sh} \varphi \\
0 & 0 & \operatorname{sh} \varphi & \operatorname{ch} \varphi
\end{array}\right]
$$

\section{ЛИТЕ РАТ У РА}

1. Кард П. Г. Анализ и синтез многослойных ннтерференционных пленок. Таллин, «Валгус», 1971.

Тартуский государственный университет
Поступила в редакцию 25/II 1985

\section{P. KARD}

\section{NEELAVATE KILEDE SELGENDAMINE}

Neelava optilise interferentskile selgendamine tähendab suhte $A / T$ minimeerimist, kus $A$ on kile neeldumistegur ja $T$ läbilaskvustegur. Selgendamiseks on vaja lisada neelava kile tagumisele poolele sobivalt valitud mitteneelav kile. Artiklis on tuletatud tingimused, mida peab rahuldama selgendav lisakile, ja selgendatud kile interferentsmaatriksi kuju. Kogu teooria aluseks on kilede interferentsmaatriksite rühma vektoresitus.

\section{|P. KARD}

\section{DIE VERGUTUNG DER ABSORBIERENDEN SCHICHTSYSTEME}

Unter der Vergütung eines absorbierenden Schichtsystems versteht man die Minimisierung des Verhältnisses $A / T$ (Absorptionsvermögen zur Durchlässigkeit des Systems). Dazu bedarf man eines zusätzlichen Schichtsystems, das hinter dem zu vergütenden System gebracht werden muB. Im Aufsatze werden die Bedingungen gefunden, denen dieses zusätzliche Schichtsystem genügen muß. Alsdann wird die Interferenzmatrix des vergüteten Systems aufgestellt. Der ganzen Theorie liegt die vektorielle Darstellung der Gruppe der Interferenzmatrizen zugrunde. 